\title{
THE MAY MEETING IN PALO aLTO
}

The four hundred ninety-second meeting of the American Mathematical Society was held at Stanford University, Palo Alto, California, on May 2, 1953. Attendance was approximately 120, including the following 88 members of the Society:

H. L. Alder, C. B. Allendoerfer, T. M. Apostol, H. M. Bacon, E. M. Beesley, Donald C. Benson, Stefan Bergman, Kurt Bing, W. W. Bledsoe, R. N. Bradt, J. L. Brenner, Paul Brock, F. H. Brownell, Herman Chernoff, Randolph Church, P. A. Clement, K. L. Cooke, E. L. Crow, P. H. Daus, A. C. Davis, E. A. Davis, C. R. DePrima, Douglas Derry, Roy Dubisch, Arthur Erdélyi, Paul Erdös, F. D. Faulkner, Solomon Feferman, J. M. G. Fell, W. J. Firey, Harley Flanders, G. E. Forsythe, A. L. Foster, Joel Franklin, K. S. Ghent, M. A. Girshick, J. W. Green, R. M. Hayes, J. G. Herriot, M. R. Hestenes, Edwin Hewitt, Jan Kalicki, William Karush, R. S. Lehman, Joseph Lehner, R. B. Leipnik, Hans Lewy, Charles Loewner, A. T. Lonseth, A. V. Martin, R. B. Merkel, A. B. Mewborn, F. R. Morris, A. P. Morse, T. S. Motzkin, A. F. Moursund, R. G. Needels, Ivan Niven, C. D. Olds, T. K. Pan, C. L. Perry, Jr., George Pólya, F. M. Pulliam, Joseph Putter, C. H. Rawlins, Jr., R. M. Redheffer, Edgar Reich, H. J. Reiter, J. B. Robinson, R. M. Robinson, E. B. Roessler, H. L. Royden, Herman Rubin, M. M. Schiffer, Abraham Seidenberg, M. A. Shader, W. H. Simons, Ernst Snapper, J. M. Stark, E. G. Straus, M. V. Sunseri, Irving Sussman, Gabor Szegö, Alfred Tarski, J. W. Weihe, Robert Weinstock, G. T. Whyburn, A. R. Williams.

The general session at ten o'clock was presided over by Professor Charles Loewner. At eleven, Professor Ernst Snapper of the University of Southern California gave the invited address, Equivalence relations in algebraic geometry. There were two sessions in the afternoon for contributed papers, at which Professors Roy Dubisch and E. M. Beesley presided.

The abstracts of papers presented at the meeting follow. Those whose numbers are followed by " $t$ " were presented by title. Professor Fraïssé was introduced by Professor Tarski and Professor Karlin by the Associate Secretary. In the absence of Mr. Vaught, paper 522 was presented by Mr. Solomon Feferman. Paper 508 was presented by Professor Straus, paper 519 by Dr. Motzkin, and paper 523 by Professor Hewitt.

\section{Algebra AND Theory of Numbers}

\section{J. L. Brenner: Orthogonal matrices of modular polynomials.}

The result of bordering a $2 \times 2$ orthogonal matrix is called a plane rotation, as usual. Theorem. Every orthogonal matrix, the elements of which are polynomials in $t$ with coefficients in the field of two elements, is a product of constant matrices and plane rotations. This theorem is false for matrices over $C_{p}[t], p>2, p$ prime [Amer. Math. Monthly vol. 58 (1951) pp. 327-329]. A whole class of nonconstant orthogonal 
$n \times n$ matrices over $C_{p}[t]$ is constructed $(n \geqq 3)$. (L. Carlitz, in an article to appear in Amer. Math. Monthly, had constructed $p \times p$ matrices of this type.) When $p>2$, it is not determined whether the construction yields all matrices $A$ such that $\operatorname{det}(E+A)=0$. (Received March 5, 1953.)

494. Roland Fraïssé: On a decomposition of relations which generalizes the sum of ordering relations.

Let $A\left(x_{1}, \cdots, x_{n}\right)$ be an $n$-ary relation with a base $E$ (a function defined on $E^{n}$, but not on the diagonal elements, assuming two values). A decomposition of $A$ is defined by a partition of $E$ into $E_{i}$ 's (with some $E_{i}$ containing at least two elements) and by a main relation $P$ with the base $\left\{E_{i}\right\}$, such that if $x_{m}(1 \leqq m \leqq n)$ are not all in the same $E_{i}$, then $A\left(x_{1}, \cdots, x_{n}\right)=P\left(E_{i_{1}}, \cdots, E_{i_{n}}\right)$ where $x_{m} \in E_{i_{m}}$. A relation is primitive if no such decomposition exists. The following are proved: (1) Given $A$ with a finite base $E$, if the main relation is primitive, then it is unique up to isomorphism. If we apply this process to each restriction of $A$ to $E_{i}$, we obtain, by iteration, a finite set $\Gamma$ of primitive relations. Any two $\Gamma$ 's obtained by different sequences of decompositions can be put into 1-1 correspondance such that the corresponding relations are isomorphic. (2) Let $\Sigma$ be the class of $n$-ary relations with a denumerable base, such that $A \in \Sigma$ iff every $n$-ary relation with a denumerable base is isomorphic to a restriction of $A$. (a) $\Sigma$ contains some primitive relations. (b) If $A \in \Sigma$, either $P \in \Sigma$ or at least one of the restrictions of $A$ to an $E_{i}$ is in $\Sigma$. (Received March 11, 1953.) I.

495t. J. R. Jackson: On the existence problem of linear programming.

This paper is concerned with the following linear programming problem: Given $m \times n$ matrix $A$ and $m \times 1$ vector $b$, does there exist $n \times 1$ vector $x \geqq 0$ such that $A x=b$ ? It is first shown that the following three conditions are equivalent: (i) there exists $m \times 1$ vector w such that $w^{\prime} A>0$ (i.e., each component of $w^{\prime} A$ is positive); (ii) if $x \geqq 0$ and $A x=0$, then $x=0$; (iii) for any $m \times 1$ vector $b$, the set $[x \mid A x=b, x \geqq 0]$ is bounded. Obviously, if $w^{\prime} A>0$, then a necessary condition for the existence of $x \geqq 0$ with $A x=b$ is that $w^{\prime} b>0$. If $w^{\prime} A>0$ and $w^{\prime} b>0$; then $w$ can be modified slightly to get $v$ with $v>0, v^{\prime} A>0$, and $v^{\prime} b>0$; and then a simple transformation of $A$ yields matrix $B$ such that each component of $v^{\prime} B$ equals $v^{\prime} b$; while the existence of $x \geqq 0$ with $A x=b$ is equivalent to the existence of $y \geqq 0$ with $B y=b$ (and, in fact, the sets of vectors $x$ and $y$ satisfying these two conditions are very simply related). Having formed matrix $H$ by subtracting $b$ from each column of $B$, the following is proved: The zerosum two-person game with matrix $H$ has non-negative value; its value is zero if and only if there exists $x \geqq 0$ with $A x=b$; moreover, if the value is zero, then the optimal mixed strategies for the second player in this game are precisely the vectors $y \geqq 0$ with $B y=b$. (Received March 4, 1953.) II.

496t. J. R. Jackson: On the existence problem of linear programming.

Under the assumption that there exists $m \times 1$ vector $w$ such that $w^{\prime} A>0$ and $w^{\prime} b>0$, it is possible to construct another game with the properties of the game $H$ of the previous abstract. Let $B^{0}$ be a matrix whose columns form a maximal linearly independent subset of the columns of $B$ (considered as vectors). Then there exists matrix $D$ such that $B=B^{0} D$. Obviously, a necessary condition for the existence of $x \geqq 0$ with $B x=b$ is that there exist vector $e$ such that $b=B^{0} e$. In this case, it is not 
difficult to see that $B x=b$ if and only if $D x=e$. It is easily seen that if $I$ is the vector of suitable dimension each of whose components is 1 , it follows that $I^{\prime} D>0$ and $I^{\prime} e>0$. Thus, the theorem of the previous abstract can be applied to the problem of whether there exists $x \geqq 0$ such that $D x=e$, with $v=I$. This result is of potential interest from a computational point of view, since $B^{0}, D$, and $e$ are easily obtained in many applications. (Received March 4, 1953.)

497. R. S. Lehman: Approximation of improper integrals by sums over multiples of irrational numbers.

Let $\alpha$ be a positive irrational number. Let $p_{i} / q_{i}(i=0,1,2, \cdots)$ be the convergents in the regular continued fraction expansion of $\alpha$. Suppose $f(x)$ is a periodic function with period 1 , improperly Riemann integrable on the interval $(0,1)$, bounded in every closed sub-interval which excludes the points 0 and 1 , and either bounded or monotone near 0 and near 1 in $(0,1)$. While Weyl's result for properly integrable $f(x)$ that $N^{-1} \sum_{n=1}^{N} f(n \alpha) \rightarrow \int_{0}^{1} f(x) d x$ as $N \rightarrow \infty$ does not apply in general, it is found that the sum does tend to the integral provided certain terms are struck out of the sum. Which terms are struck out does not depend on the particular function $f(x)$ but only on the arithmetic character of $\alpha$. More precisely, let $q_{i} \leqq N<q_{i+1}$ so that $N=b q_{i}$ $+r, q_{i}>r \geqq 0$; then the omitted terms are those for which $n=k q_{i}(k=1,2, \cdots, b)$. It is shown that cancelling terms from the sum in the above approximation formula becomes necessary only for a set of $\alpha$ having measure zero; in particular it is unnecessary for $\alpha$ having continued fractions with bounded quotients. (Received March 11, 1953.)

\section{8t. R. D. Stalley: $A$ new number density.}

The density of a sequence of positive integers $n_{1}<n_{2}<\cdots$ is defined to be glb $k / n_{k}$. Two theorems concerning the densities $\alpha, \beta$, and $\gamma$ of sequences $A, B$, and $C=A+B$ are proved. First, if $\alpha+\beta>1$, then $\gamma=1$. Second, if $1,2, \cdots, k \in A$, then $\gamma \geqq k(k+1)^{-1} \alpha+\beta$ or $\gamma=1$, where this is a best possible result. Since this density is a modified Schnirelmann density, it is unexpected that these results are identical to known results for asymptotic density. (Received March 9, 1953.)

\section{9t. J. D. Swift: Some remarks on the numbers of Chernick and Carmichael.}

A Chernick number is defined as a product $n_{1} n_{2} n_{3}=N$ such that $N \equiv 1 \bmod \left(n_{i}-1\right)$, $i=1,2$, 3. In Bull. Amer. Math. Soc. vol. 45, pp. 269-273, J. Chernick showed that a sufficient condition that $N$ be an absolute pseudo-prime or Carmichael number is that $n_{1}, n_{2}$, and $n_{3}$ be prime. This condition is not necessary. On the other hand, Carmichael numbers exist which are not Chernick numbers. For example, a Carmichael number with four prime factors $p, q, r, s$ is a Chernick number if and only if at least one of the relations of the type $p q-1$ divides $r s-1$ holds. If the quotient of the last named numbers is $k$, a Chernick number $(p q) \cdot r \cdot s$ is an absolute pseudoprime if $p-1$ divides $(k q+1)(q-1)$ and the similar relation holds with $p$ and $q$ interchanged. The tests may be extended to more complicated cases and applied to the construction of Carmichael numbers. Similar considerations apply to the pseudoprime character relative to particular bases such as 2. (Received March 12,1953.)

500. Alfred Tarski: Universal arithmetical classes of mathematical systems. Preliminary report. 
For notation see Tarski, Proceedings of the International Congress of Mathematicians, 1950, vol. 1, p. $705 \mathrm{ff}$. Consider systems $\mathfrak{A}, \mathfrak{B}, \cdots$ formed by nonempty sets $A, B, \cdots$ and binary relations $R \subseteq A \times A, S \subseteq B \times B, \cdots$. $\cong \mathbb{B}$ means that $\mathfrak{A}$ and $\mathfrak{B}$ are isomorphic; $\mathfrak{A} \subseteq \mathfrak{B}$ means that $\mathfrak{A}$ is a $\mathfrak{B}$-subsystem, i.e., $A \subseteq B$ and $R$ $=S \cap(A \times A)$. $\delta$ being a set of systems, the meaning of formulas $S \in A C, S \in A C_{\delta}$ is known. $S \in U C$ means that $S$ is a universal arithmetical class, i.e., $\mathcal{S}$ consists of all systems satisfying a first-order sentence of the form $\prod_{x}, \ldots \phi(x, \cdots)$ where $\Pi$ is the universal quantifier and $\phi(x, \cdots)$ a formula without quantifiers. $S \in U C_{\delta}$ means that $\mathcal{S}$ consists of all systems satisfying a certain set of such sentences. Theorems: $I$. If $\mathfrak{A}$ is a finite and $\mathcal{S}$ consists of all $\mathfrak{B}$ 's such that $\mathfrak{A} \cong \mathfrak{Y}^{\prime}$ for no $\mathfrak{X}^{\prime} \subseteq \mathfrak{Q}$, then $S \in U C$. II. $S \in U C_{\delta}$ if and only if $\mathfrak{B} \in \mathcal{S}$ whenever (i) $\mathfrak{B} \cong \mathfrak{A}$ or $\mathfrak{B} \subseteq \mathfrak{A}$ for some $\mathfrak{A} \in \mathcal{S}$, or (ii) $\mathcal{S}$ contains all finite $\mathfrak{B}$-subsystems. III. $\mathcal{S} \in U C$ (or $\mathcal{S} \in U C_{\delta}$ ) if and only if $S \in A C$ (or $S \in A C_{\delta}$, respectively), and $\mathfrak{B} \in \mathcal{S}$ whenever $\mathfrak{B} \subseteq \mathfrak{A}$ for some $\mathfrak{A} \in \mathcal{S}$. I-III extend to arbitrary systems with finitely many relations and operations of finite ranks. (Received March 11, 1953.)

501t. R. L. Vaught: Remarks on universal arithmetical classes. Preliminary report.

For notation see the preceding abstract of Tarski. The following theorem analogous to Theorem II of that abstract holds: $\mathcal{E} \in U C$ if and only if (i) $\mathscr{B} \in S$ whenever $\mathscr{B} \cong \mathfrak{A}$ or $\mathfrak{B} \subseteq \mathfrak{A}$ for some $\mathfrak{A} \in S$, and (ii) there is a natural number $n$ such that $\mathfrak{B} \in \mathcal{S}$ whenever $\mathcal{S}$ contains all $\mathbb{B}$-subsystems with at most $n$ elements. Neither this theorem nor Theorems I and II of the preceding abstract apply in general to systems with infinitely many relations and operations of finite ranks. (However, as was remarked by Tarski, his Theorem III applies to arbitrary such systems. In this case the formula $S \in A C_{\delta}$ is understood to mean that $S$ is an intersection of arbitrarily many sets $\tau \in A C$, and similarly for $\delta \in U C_{\delta .}$ ) (Received March 11, 1953.)

\section{ANALYsis}

502. F. H. Brownell: Flows and noncommuting projections on Hilbert space.

Let $\{E(A)\}$ over Borel subsets $A$ of the real line be a resolution of the identity for the Hilbert space $X$ and consider the flow $u_{t}=U_{t} u_{0}=\int_{-\infty}^{\infty} e^{i t \lambda} d E(\lambda) u_{0}$ for a fixed $u_{0} \in X$ and $t$ real, the integral existing as the $X$ norm limit of the Lebesgue-Stieltjes sums. Let $P$ be a projection in $X$, not commuting with $E(A)$ in the nontrivial case. The problem is to study the asymptotic behavior of $\left\|P u_{t}\right\|^{2}=\left(P u_{t}, u_{t}\right)$ as $t \rightarrow+\infty$. If $P$ is compact (i.e. finite-dimensional), it is possible to shift the integration so that $\left\|P u_{t}\right\|^{2}=\int_{-\infty}^{\infty} e^{i s t} d \psi(s)$, where $\psi(A)=\int_{-\infty}^{\infty} \int_{-\infty}^{\infty} \chi_{A}(\lambda-\beta) d_{\lambda} d_{\beta}\left(P E(\lambda) u_{0}, E(\beta) u_{0}\right)$ suitably defined is $\sigma$-additive and of bounded variation over the Borel sets of $(-\infty, \infty)$. Thus it is known that $\left\|P u_{t}\right\|^{2}$ converges densely to a constant $C$ if and only if $\psi(\{s\})$ $=0$ for all real $s \neq 00$, and in this case $C=\psi(\{0\})$. This condition on $P$ and the extension to noncompact $P$ are studied. (Received March 13, 1953.)

\section{3t. H. E. Chrestenson: A class of generalized Walsh functions.}

For each integer $\alpha \geqq 2$ the system $\Psi_{\alpha}=\left\{\psi_{j}(x)\right\}(j=0,1, \cdots)$ of Walsh functions of order $\alpha$ is defined. Each $\Psi_{\alpha}$ is an orthonormal system, complete in $L(0,1)$. Let $f(x)$ be integrable and periodic, and let the Fourier series for $f(x)$ be $\sum_{n=0}^{\infty} c_{n} \psi_{n}(x)$. Write $s_{n}(x)=\sum_{r=0}^{n-1} c_{n} \psi_{n}(x)$. The following results are proved: (i) As $n \rightarrow \infty, s_{\alpha^{n}}(x) \rightarrow f(x)$ a.e. (ii) If $f(x)$ has total variation $V<\infty$, then $s_{n}(x) \rightarrow f(x)$ a.e., and $\left|c_{n}\right| \leqq V k^{-1} \csc \pi / \alpha$. 
(iii) If $[f(t)-c]\left[t-x_{0}\right]^{-1} \in L\left(x_{0}-\delta, x_{0}+\delta\right)$ for some $\delta>0$, then $s_{n}\left(x_{0}\right) \rightarrow c$. (iv) If the modulus of continuity of $f(x)$ satisfies $\omega(\delta ; f)=o\left(\left(\log \delta^{-1}\right)^{-1}\right)$ as $\delta \rightarrow 0$, then $s_{n}(x) \rightarrow f(x)$ uniformly. (v) If $f(x)$ is continuous, then $\sum_{n=0}^{\infty} c_{n} \psi_{n}(x)$ is uniformly $(C, 1)$ summable to $f(x)$. These results include some of those of N. J. Fine (Trans. Amer. Math. Soc. vol. 65 (1949) pp. 372-414) and J. L. Walsh (Amer. J. Math. vol. 55 (1923) pp. 5-24). (Received March 9, 1953.)

\section{4t. H. E. Chrestenson: Some groups of orthonormal functions.}

Let $\Lambda=\left\{\lambda_{j}(x)\right\}(j=0,1, \cdots)$ be a set of complex-valued orthonormal functions on $(0,1)$. If for each $j$ and $k, \lambda_{j}(x) \bar{\lambda}_{k}(x) \in \Lambda$, then $\Lambda$ is a multiplicative group. For each integer $\alpha \geqq 2$, a system $\Psi_{\alpha}=\left\{\psi_{j}(x)\right\}$ is defined so that each $\psi_{j}(x)$ has order dividing $\alpha$. $\Psi_{2}$ is the system of Walsh functions. Suppose that the group $\Lambda$ is isomorphic to $\Psi_{\alpha}$ so that $\lambda_{j}(x)$ and $\psi_{j}(x)$ correspond. Necessary and sufficient conditions are given for the existence of a measurable transformation $T x$ having the properties (i) for any measurable set $E \subset[0,1), \mu\left(T^{-1} E\right)=\mu(E)$, and (ii) for all $n \geqq 0, \lambda_{n}(x)=\psi_{n}(T x)$ a.e. These results include those of Paul Civin (Pacific Journal of Mathematics vol. 2 (1952) pp. 291-295) for $\alpha=2$ and $\lambda_{n}(x)$ real-valued. (Received March 9, 1953.)

\section{5t. Paul Civin: Orthonormal cyclic groups.}

Let $\left\{A_{n}(x)\right\}(n=0, \pm 1, \cdots ; 0 \leqq x \leqq 1)$ be a set of complex measurable functions which is a multiplicative cyclic group. A necessary and sufficient condition that $\left\{A_{n}(x)\right\}$ be an orthonormal system over $0 \leqq x \leqq 1$ is that the generator of the group admit a representation exp $(2 \pi i c(x))$ almost everywhere, with $c(x)$ equimeasurable with $x$. A similar result relating real functions such that $A_{n}^{2}(x)=1$ and the Walsh functions is contained in a note of the authors in the Pacific Journal of Mathematics vol. 2 (1952) pp. 291-296. (Received March 9, 1953.)

506. P. A. Clement: Generalized convexity and inequalities im grossen of functions on surfaces of negative curvature.

Certain explicit functions involving the circumference $l(r)$ and area $a(r)$ of a geodesic circle on an analytic surface whose Gaussian curvature $K$ satisfies $K \leqq K_{0}<0$ $\left(K_{0}=\right.$ constant $)$ are shown to satisfy a Condition $\mathrm{C}$ : they are subfunctions of the twoparameter family of solutions of the second order differential equation $\left(D^{2}+K_{0}\right) u=0$, zero at the pole, monotonic nondecreasing, and identically zero if and only if $K \equiv K_{0}$. A function $f(r)$ is shown to be a subfunction of the family by verifying that $f(r)$ satisfies the defining functional inequality, or, if $f(r)$ is of class $C^{2}$, that it satisfies a differential inequality [see Peixoto, Generalized convex functions and second order differential inequalities, Bull. Amer. Math. Soc. vol. 55 (1949) pp. 563-572]. For example, the isoperimetric function, $l^{2}(r)+K_{0} a^{2}(r)-2 \pi a(r)$, satisfies Condition C, as do also a number of functions obtained from it by a "modification" process. The inequality implied for a function satisfying Condition $\mathrm{C}$ characterizes the surface for some functions. Other configurations than geodesic circles are considered and some of the functions retain characteristic properties on generalization; for example, some functions which are generalizations of Steiner's formulas are shown to satisfy a generalized Condition C. (Received March 10, 1953.)

507. K. L. Cooke: The rate of increase of real continuous solutions of algebraic differential-difference equations of the first order.

In this paper, bounds are established on the possible rate of growth, as $t \rightarrow+\infty$, 
of real continuous solutions of algebraic differential-difference equations of the form $P\left(t, u(t), u^{\prime}(t), u(t+1), u^{\prime}(t+1)\right)=0$, where $t$ is a real variable and where $P(t, u, v, w, x)$ is a polynomial, with real coefficients, in the variables $t, u, v, w, x$. The paper extends previous work by Borel (Ann. Ecole Norm. vol. 16 (1899) pp. 9-136) and Shah (Bull. Amer. Math. Soc. vol. 53 (1947) pp. 548-558), who discussed the analogous problem for algebraic differential equations and for algebraic difference equations, respectively. The methods used here are similar to those of Borel and Shah. A typical theorem is the following: To each polynomial $P(t, u, v)$ there corresponds a positive number $A$ with the property that if $u(t)$ is a real solution of $P\left(t, u(t), u^{\prime}(t+1)\right)=0$ which exists and has a continuous first derivative for $t \geqq t_{0}$, then there is no number $T>0$ for which $|u(t)| \geqq e_{2}(A t)$ for all $t \geqq T$. Stronger theorems can be proved if, and only if, the differential-difference equations are of more restricted type. (Received March 9, 1953.)

\section{Paul Erdös and E. G. Straus: Linear independence of sequences in a Banach space.}

The following theorem is proved: Let $x_{1}, x_{2}, \cdots$ be a sequence of unit vectors in a Banach space such that every finite subset is linearly independent, and let $M_{1}, M_{2}, \ldots$ be a sequence of non-negative numbers. Then there exists a subsequence $\left\{x_{i_{n}}\right\}$ with the property that $\lim _{m \rightarrow \infty} \sum_{n} c_{n m} x_{i_{n}}=0$ and $\left|c_{n m}\right| \leqq M_{n}$ implies $\lim _{n \rightarrow \infty} c_{n m}=0$ for all $n$. (Received March 11, 1953.)

509. G. E. Forsythe: Arbitrarily close lower bounds for the fundamental frequency of certain vibrating membranes.

Divide the plane into equal squares $S_{i}$ of side $h$ by a network of parallels to the axes. A "half-square" is an isosceles right triangle which is half an $S_{i}$. Let the fixed convex open region $R$ with boundary $C$ be a finite union of squares and half-squares, for all $h$ under consideration. Let $\lambda$ be the least number such that $\left({ }^{*}\right) u_{x x}+u_{y y}+\lambda u=0$ in $R$ and $u=0$ on $C$. (Subscripts denote differentiation.) Let $\lambda_{h}$ be the least number such that $v(x+h, y)+v(x-h, y)+v(x, y+h)+v(x, y-h)-4 v(x, y)+\lambda_{h} v(x, y)=0$ at the network nodes $(x, y)$ in $R$, and $v=0$ at the nodes on $C$. Theorem: For all $h$ under consideration, as $h \rightarrow 0$ and $a=a(R)=\iint_{R}\left(u_{x x}^{2}+u_{y y}^{2}\right) d x d y / \iint_{R}\left(u_{x}^{2}+u_{y}^{2}\right) d x d y$, where $u$ solves $(*), \lambda_{h} / \lambda \leqq 1-a h^{2} / 12+o\left(h^{2}\right)$. Application: for all sufficiently small $h$ the ordinary finite-difference approximation $\lambda_{h}$ is a rigorous lower bound for $\lambda$. Since $\lambda_{h} \rightarrow \lambda$ as $h \rightarrow 0$, for all sufficiently small $h$ these $\lambda_{h}$ can be teamed with upper bounds from other methods [e.g., Rayleigh-Ritz; or Pólya, C. R. Acad. Sci. Paris vol. 235 (1952) pp. 995-997] to bound $\lambda$ arbitrarily closely. In proving the theorem one sets the $u$ solving $\left(^{*}\right)$ into the Rayleigh quotient of quadratic forms, $N(v) / D(v)$, whose minimum is $\lambda_{h}$, and compares $N(u) / D(u)$ with $-\iint_{R} u\left(u_{x x}+u_{y y}\right) d x d y / \iint_{R} u^{2} d x d y=\lambda$. (Received March 9, 1953.)

\section{Samuel Karlin: On the renewal equation.}

A unified approach to the renewal equation $u(x)=\int_{-\infty}^{\infty} u(x-t) d F(t)+G(x)$ allowing negative arguments is presented. Only special examples of this general case have been treated thus far. This includes both the discrete and continuous equations. All the asymptotic relations are reduced to the general Wiener Tauberian theorem. Various rates of convergence of $\lim _{x \rightarrow \infty} u(x)$ and $\lim _{x \rightarrow-\infty} u(x)$ are obtained by assuming existence of higher moments of $F$ and $G$. This presentation includes as special cases the recent results of papers of Chung and Wolfowitz (Ann. of Math. (1952)) and Chung and Pollard (Proc. Amer. Math. Soc. (1952)). Finally, limiting theorems 
for the renewal equations in higher dimensions are obtained and the treatment is generalized to include renewal type theorems involving groups and semi-groups. (Received April 13, 1953.)

\section{Joseph Lehner: Note on the Schwarz triangle functions.}

The triangle functions referred to are those that map a certain curvilinear triangle on a half-plane; they are automorphic on the group $\Gamma(\lambda)$ generated by $S: z \rightarrow z+\lambda$, $T: z \rightarrow-1 / z$, where $\lambda$ takes one of the values $\lambda=2 \cos \pi / q, q=3,4,5, \cdots$ If $\phi_{\lambda}(z)$ is a triangle function, it will have a simple pole at $z=i \infty$ and be otherwise regular. We normalize $\phi_{\lambda}(z)=x^{-1}+\sum_{n=0}^{\infty} c_{n}(\lambda) x^{n}$, where $x=\exp 2 \pi i z / \lambda$. We prove: I. For every $\lambda$ and every $n>0, c_{n}(\lambda)$ is a rational number. II. $c_{n}(\lambda) \sim(2 \lambda)^{-1 / 2} \cdot n^{-3 / 4} \cdot \exp 4 \pi n^{1 / 2} / \lambda$, as $n \rightarrow \infty$. I is proved by considering the inverse of $\phi$, which is known to be the quotient of two solutions of the hypergeometric differential equation (with parameters depending on $\lambda$ ). II follows from the Hardy circle method, where only the largest arc in the dissection of the circle is treated. (Received February 27, 1953.)

\section{R. B. Leipnik: Heaviside calculus of abstract power series.}

The notation of a powered set is defined. The group algebra of unconditionally convergent power series over a powered set is shown to be a powered set by validating some formal manipulations due to Heaviside. Applications to Heaviside calculus are obtained. (Received March 12, 1953.)

513t. B. L. McAllister and C. J. Thorne: Reverse derivatives and reverse differential equations.

A formula for the $n$th derivative of $y$ with respect to $x$ is given as a function of derivatives of $x$ with respect to $y$. If, in a solvable differential equation of order 2 or larger in $y$, the derivatives are replaced by reverse derivatives, the new differential equation in $x$ has the same solution, in general. The new differential equation is usually nonlinear. The use of this method on the nonlinear differential equations presently in the literature usually leads to a form of Abel's equation. Occasionally this leads to solutions; more of ten, it shows that the solution to the nonlinear differential equation has movable singularities. That is, the singularities are functions of the initial conditions. (The results presented in this paper were obtained in carrying out Contract No. N7-ONR-45107 between the office of Naval Research and the University of Utah.) (Received March 2, 1953.)

514t. T. S. Motzkin and J. L. Walsh: Least pth power polynomials on a complex finite point set.

Let $E$ be the set of $m$ distinct complex points $\left\{z_{1}, z_{2}, \cdots, z_{m}\right\}$, and for variable $\zeta_{i}$ let the polynomial $T_{n}(z) \equiv \prod_{1}^{n}\left(z-\zeta_{i}\right)$ minimize $\sum_{1}^{m} \mu_{i}\left|T_{n}\left(z_{i}\right)\right| p, \mu_{i}>0,0<n<m$. Let any polynomial with no zero on $E$ be called proper. Let $S_{n}(z) \equiv z^{n}+\cdots$ denote a ("stationary") polynomial such that $\sum_{1}^{m} \mu_{i}\left|S_{n}\left(z_{i}\right)\right|^{p-2} S_{n}\left(z_{i}\right) \bar{u}\left(z_{i}\right)=0$ for every polynomial $u(z)$ of degree $n-1$. For each $p>0$ and arbitrary $\mu_{i}$ the set of proper stationary polynomials is precisely the set of proper extremal polynomials in the sense of Fekete (1951) $(p=\infty)$. For $p>1$ and given $\mu_{i}$ the polynomials $S_{n}(z)$ and $T_{n}(z)$ are unique and coincide. For $p \leqq 1$ and $\mu_{1}>\sum_{2}^{m} \mu_{i}$, every $T_{n}(z)$ has a zero in $z_{1}$. For $p=1$ and given $\mu_{i}$ the $T_{n}(z)$ form in the $2 n$-space of coefficients a convex polyhedron $P$ of dimension $s \leqq n$. For $s=n$ and noncollinear $z_{i}, P$ is a simplex. If a proper $T_{n}(z)$ (or equivalently $\left.S_{n}(z)\right)$ exists, then $s \geqq 2 n-m+1$. For $n=m-1$, set $\omega(z) \equiv \prod_{1}^{m}\left(z-z_{i}\right)$ 
and suppose $\quad \mu_{1}\left|\omega^{\prime}\left(z_{1}\right)\right|=\mu_{2}\left|\omega^{\prime}\left(z_{2}\right)\right|=\cdots=\mu_{r}\left|\omega^{\prime}\left(z_{r}\right)\right|<\mu_{r+1}\left|\omega^{\prime}\left(z_{r+1}\right)\right| \leqq \cdots$ $\leqq \mu_{m}\left|\omega^{\prime}\left(z_{m}\right)\right|$; then $P$ is precisely the simplex $\omega(z) \sum_{1}^{\prime} \lambda_{i} /\left(z-z_{i}\right), \quad \sum \lambda_{i}=1, \lambda_{i} \geqq 0$. (Received December 9, 1952.)

\section{R. M. Redheffer: Even canonical products.}

Let $F(z)=\Pi\left(1-z^{2} / \lambda_{n}^{2}\right), \lambda_{n}>0, \Lambda(u)=$ number of $\lambda^{\prime}$ s less than $u$. Then the following statements are equivalent: (a) $\lim \sup \log |F(x)| / x=0$ and $\lim \sup \Lambda(u) / u$ $=D<\infty$; (b) $\lim \sup \log \left|F\left(r e^{i \theta}\right)\right| / r=\pi D^{\prime}|\sin \theta|$ for some $D^{\prime}<\infty$ and all $\theta$. Also the following statements are equivalent: (a) $\lim \Lambda(u) / u=D$; (b) Given $\epsilon>0$ there is a constant $C=C(\epsilon)$ such that for $\pi-\epsilon \geqq|\theta| \geqq \epsilon$ one has $\pi D r|\sin \theta|-\epsilon r \leqq \log \left|F\left(r e^{i \theta}\right)\right|$ $\leqq \pi D r|\sin \theta|+\epsilon r$ whenever $r>C$. The latter generalizes to complex $\lambda_{n}$, to give the following: Let $F(z)=\prod\left(1-z^{2} / \lambda_{n}^{2}\right), \lim n / \lambda_{n}=D$, let $G(z)=\prod\left(1-z^{2} / \mu_{n}^{2}\right), \lim n / \mu_{n}$ $=D^{\prime} \neq D$. Then the roots $\pm z_{n}$ of $F(z)=G(z)$ satisfy $\lim n / z_{n}=\max \left(D, D^{\prime}\right)$. (Received February 12, 1953.)

\section{Edgar Reich: An inequality for subordinate analytic functions.}

Let $f(z)$ and $F(z)$ be nonconstant analytic functions, regular in the unit circle, $|z|<1$, and suppose that $f$ is subordinate to $F$ (J. E. Littlewood, Lectures on the theory of functions, London, 1944, pp. 163-171). Let $a(r)$ and $A(r)$ be the areas of the regions on the Riemann surfaces onto which the disk $|z|<r$ is mapped by $f$ and $F$ respectively. It is shown that $a(r) / A(r) \leqq \max _{k=1,2,}, \cdots\left(k r^{2 k-2}\right)(0<r<1)$, where the inequality is the best possible one in the sense that for any fixed $r$ in the range $(0,1)$ there exist functions $f(z)$ and $F(z)$ satisfying the hypotheses, such that the inequality becomes an equality. The above result supplements one of G. M. Goluzin [Mat. Sbornik N.S. vol. 29 (1951) pp. 209-224]. (Received March 20, 1953.)

\section{Applied Mathematics}

517t. K. S. Miller and R. J. Schwarz: On the interference of pulse trains.

In various electronic applications one frequently has to deal with trains of periodic pulses. This paper considers the problem of determining explicit formulas for the coincidence time of two or more pulse trains of arbitrary period and pulse width. Formulas for the fraction of time two pulse trains (with arbitrary initial phase) are coincident for a duration greater than a prescribed length of time are also deduced. Multiple pulse trains are briefly considered. Number theoretic methods are employed. (Received March 23, 1953.)

\section{GEOMETRY}

518. Douglas Derry: On differentiable closed curves of order $n$ in n-space.

Let $C_{n}$ be a closed curve in real projective $n$-space for which osculating $k$-spaces $(k, s), 0 \leqq k \leqq n-1$, are defined for every curve point $s$ of $C_{n}$. Those $n-2$-spaces $L$ which contain no points within $n$ distinct $(n-1, s)$ are shown to be exactly those $n-2$-spaces $L$ for which every hyperplane through $L$ cuts $C_{n}$ in $n$ points. A characterization of such $n-2$-spaces is given. The result is applied to obtain characterizations of those points of the projective space through which less than $n(n-1, s)$ pass. (Received March 9, 1953.) 
519. T. S. Motzkin and C. B. Tompkins: Boundedness of sequential projections. Preliminary report.

A convex set $S$ is polyhedral at infinity if $S$ is the union of a bounded convex set and of the intersection of a finite number of half-spaces. It is shown that for finitely many closed convex sets $S_{i}$ in an inner-product space that are polyhedral at infinity there exists a nonvoid bounded closed convex set $C$ such that for every point $P$ of $C$ also the projection $P_{i}$ of $P$ on $S_{i}$ (that is, the nearest point of $S_{i}$ to $P$ ) belongs to $C$ for each $i$. It follows that the set of all points obtained from a given point $P$ by projection on some $S_{i_{1}}$, from the resulting point on $S_{i_{2}}$, and so on, is bounded. The same is true if, in all former statements, $P_{i}$ is replaced by $P+\lambda\left(P_{i}-P\right), 0 \leqq \lambda<2$. Applications are given to the solutions of systems of linear equations and inequalities. The theorems subsist for general strictly convex Banach spaces of dimension 2, but not of higher dimension. (Received May 1, 1953.)

520. T. K. Pan: Relative first curvature and relative parallelism in a subspace of a Riemannian space.

Let $V_{n}$ be a subspace of a Riemannian space $V_{m}$. Associate to each point of $V_{n}$ a set of $m-n$ arbitrary independent unit vectors in $V_{m}$, which are functions of the point and the direction at the point of $V_{n}$ and which are not in $V_{n}$ except in the asymptotic directions of $V_{n}$. With respect to these vectors, relative first curvature, relative associate curvature, pseudogeodesics and relative parallelism are defined in $V_{n}$ in $V_{m}$ to generalize the concepts and results obtained by the author in a hypersurface (Bull. Amer. Math. Soc. Abstract 58-6-699). (Received March 16, 1953.)

\section{LOGIC AND FOUNDATIONS}

521. Kurt Bing: Definability in the theory of integers. Preliminary report.

The following theorems are proved: Addition and multiplication of integers are definable in terms of lower predicate calculus without identity and a single symmetric dyadic relation. Addition and multiplication of integers are not each definable in terms of lower predicate calculus (or even second order predicate calculus) and monadic relations. For each sequence of relations of integers there exists a single symmetric dyadic relation $H$ of integers such that each relation of the sequence is definable in terms of lower predicate calculus without identity and $H$. Addition and multiplication of integers are definable in terms of a single operator and a single symmetric dyadic relation. These results are extensions, to the theory of integers, of theorems proved by Church and Quine (J. Symbolic Logic vol. 17 (1952) p. 186), Craig and Quine (ibid. p. 188), and Myhill (J. Symbolic Logic vol. 15 (1950) p. 130) for the theory of natural numbers. (Received March 11, 1953.)

522. R. L. Vaught: Applications of the generalized Skolem-Löwenheim theorem to problems of completeness and decidability.

For terminology see Tarski, Proceedings of the International Congress of Mathematicians, 1950 , vol. 1, pp. 705 ff. The following theorem easily follows from the generalized Skolem-Löwenheim theorem (stated in op. cit., Theorem 23): If $K$ is a non-empty arithmetically closed class of algebras (with at most denumerably many relations and operations) such that for some infinite power, all members of $K$ with 
that power are isomorphic, then all members of $K$ are arithmetically equivalent. A metamathematical consequence of this result is: If $T$ is a consistent theory formalized within first order logic such that every model of $T$ is infinite, and for some infinite power, all models of that power are isomorphic, then $T$ is complete; if, moreover, $T$ has a general recursive axiom system, then $T$ is decidable. This immediately implies the completenes and decidability of various theories for which these results were originally established by other methods. Examples: the theories of densely ordered sets without end points, of atomless Boolean algebras, of infinitely divisible torsionfree Abelian groups, of Abelian groups with all elements of a fixed prime order, and of algebraically closed fields. The ordinary Skolem-Löwenheim theorem (asserting the existence of countable models) was previously used in completeness proofs by A. Robinson and Tarski. (Received March 11, 1953.)

\section{Statistics and Probability}

\section{Edwin Hewitt and L. J. Savage: Symmetric measures on Car-} tesian products.

Two theorems of B. de Finetti (Ann. Inst. H. Poincare vol. 7 (1937) pp. 1-68) are generalized as follows: Let $X$ be a compact Hausdorff space, and let $Q$ be the Cartesian product of $\boldsymbol{\aleph}_{0}$ replicas of $X$. For $\phi$ a countably additive probability measure on $X$, let $\pi_{\phi}$ denote the product measure on $Q$ obtained by taking each factor-measure equal to $\phi$. Let $\psi$ be an arbitrary countably additive probability measure on $Q$ invariant under all permutations of a finite number of co-ordinates. Then there exists a Borel measure $\Psi$ on the space II of all measures $\pi_{\phi}$ (which is made into a compact Hausdorff space in a certain way) such that $\psi=\int_{\Pi} \phi_{\pi} d \Psi\left(\phi_{\pi}\right)$, the integral being of Gel'fand-Pettis type. Uniqueness is obtained under certain additional assumptions. Related theorems are proved for locally compact $X$ and for general measurable spaces $X$, with finitely additive measures. (Received March 13, 1953.)

\section{TOPOLOGY}

\section{4t. C. E. Burgess: Collections and sequences of continua in the plane.}

The following theorems are proved. (1) If, in the plane, $W$ is a collection consisting of $n$ mutually exclusive bounded continua and $G$ is a collection consisting of $n^{2}-n+1$ mutually exclusive continua each intersecting every set of $W$, then some continuum of $G$ contains a bounded continuum which intersects every continuum of $W$. (2) If $\left\{M_{n}\right\}$ is a sequence of mutually exclusive nondegenerate continua in the plane converging to a continuum $M$, then there is a sequence $\left\{T_{n}\right\}$ of bounded continua converging to $M$ such that for each $n, T_{n}$ is a subset of $M_{n}$ and is irreducible between some two points. This generalizes a result previously obtained by the author [Continua and their complementary domains in the plane, Duke Math. J. vol. 18 (1951) pp. 901-917, Theorem 5]. (3) Let $K$ be a continuum having the property that for some positive integer $n$ there exist $n$ domains intersecting $K$ such that no bounded subcontinuum of $K$ intersects each of these domains. The plane does not contain uncountably many mutually exclusive continua such as $K$. (Received March 6, 1953.)

525. A. V. Martin: Decompositions and quasi-compact mappings.

A decomposition of a topological space $M$ is any collection $G$ of nonempty, pair- 
wise-disjoint sets (not necessarily closed) of $M$ whose union is $M$. The hyperspace determined by $G$ is defined in the natural way. It is easy to prove that a topological space $X$ is the image of $M$ under a quasi-compact mapping (for definition see Whyburn, Open and closed mappings, Duke Math. J. vol. 17 (1950) pp. 69-74) if and only if $X$ is homeomorphic to the hyperspace of some decomposition of $M$. Let $M$ be a separable metric space and let $f$ be a quasi-compact mapping of $M$ onto a topological space $X$. The following results are proved: If $M$ is locally compact and $X$ is metric, then $X$ is separable and locally compact. If $f$ is monotone and $M$ is locally compact and $X$ is Hausdorff, then $X$ is separable, metric, and locally compact. If $f$ is closed, and if for every $x$ in $X, f^{-1}(x)$ is compact, then $X$ is separable and metric. (Received March 16, 1953.)

J. W. Green, Associate Secretary 